\title{
DNA methylation inhibitor, decitabine, promotes MGC803 gastric cancer cell migration and invasion via the upregulation of NEDD4-1
}

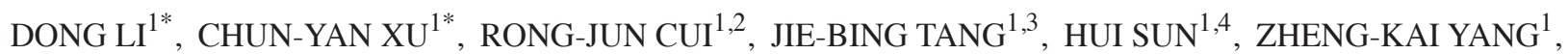 \\ JING-YI BU ${ }^{1}$, PING LIN ${ }^{1}$, NING HUANG ${ }^{1}$, YAN-DAN DU ${ }^{4}$ and XIAO-GUANG YU ${ }^{1}$
}

\author{
${ }^{1}$ Department of Biochemistry and Molecular Biology, Harbin Medical University, Harbin, Heilongjiang 150081; \\ ${ }^{2}$ Department of Biochemistry and Molecular Biology, Mudanjiang Medical University, Mudanjiang, Heilongjiang 157000; \\ ${ }^{3}$ Department of Clinical Oncology, Harbin Medical University Cancer Hospital, Harbin, Heilongjiang 150081; \\ ${ }^{4}$ Department of Clinical Laboratory, The Second Clinical Medical School of Inner Mongolia University for The Nationalities, \\ (Inner Mongolia Forestry General Hospital), Hulunbuir, Inner Mongolia 022150, P.R. China
}

Received December 15, 2014; Accepted September 9, 2015

DOI: $10.3892 / \mathrm{mmr} .2015 .4424$

\begin{abstract}
Gastric cancer is the fourth most common cancer type and the second leading cause of cancer-associated mortality worldwide. Metastasis is a crucial feature of its progression. DNA methylation provides a key epigenetic signature in the epigenetic regulation pathway, and is implicated in transcriptional regulation. $\mathrm{CpG}$ sites, which are associated with gene transcriptional activity, are underrepresented in the mammalian genome and tend to be clustered within $\mathrm{CpG}$ islands (CGIs) located in the vicinity of the transcription start sites of the majority of the protein-coding genes in humans. The DNA methylation inhibitor, decitabine (DAC), has been demonstrated to be active in hematological disorders. The majority of previous studies in cancer cells demonstrated that DAC inhibits cell proliferation and the motility of the cells. However, since demethylation across the entire genome alters the expression of a large number of genes, the effects of DAC in different tumor cell types are difficult to accurately predict. Neural precursor cell-expressed, developmentally downregulated (NEDD)4-1, a member of the NEDD4 family, which belongs to the E3-ubiquitin ligase family, was reported to be highly expressed in a wide range of tumor types, and it activates the phosphoinositide 3-kinase/Akt pathway by degrading phosphatase and tensin homolog. NEDD4-1 promotes the
\end{abstract}

Correspondence to: Professor Xiao-Guang Yu, Department of Biochemistry and Molecular Biology, Harbin Medical University, 157 Baojian Road, Harbin, Heilongjiang 150081, P.R. China

E-mail: xiaoguang_yu@hotmail.com

*Contributed equally

Key words: NEDD4, gastric cancer, decitabine, DNA methylation, MGC803, CNrasGEF migration and invasion of glioma cells via the ubiquitination and subsequent degradation of cyclic nucleotide-Ras guanine nucleotide exchange factors (CNrasGEFs). In gastric cardia adenocarcinoma, NEDD4-1 acts as an exceptional prognostic biomarker. In the present study, DAC was revealed to promote the invasive properties of MGC803 gastric cancer cells. NEDD4-1 targeted the CNrasGEF-mediated DAC invasion-promoting activity in MGC803 cells, and CGI methylation in neither the NEDD4 promoter nor the first intron was demonstrated to be associated with this effect. The results of the present study revealed that DAC exerts variable effects in different gastric cancer cell lines and may provide a reference for DAC administration in the clinic.

\section{Introduction}

Gastric cancer is the fourth most common cancer type and the second leading cause of cancer-associated mortality, with a mortality rate of $>700,000$ individuals reported annually (1). Currently, the highest incidence rates are reported in China, Japan, Korea, Eastern Europe and parts of Central and South America (2). Metastasis and post-surgery recurrence rates in gastric cancer are $>40 \%$, and therefore how to effectively deal with metastasis is a major challenge in gastric cancer therapy (3).

DNA methylation is an essential feature of the epigenetic regulation pathway, which is frequently perturbed in human gastric cancer, and acts as a key epigenetic signature implicated in transcriptional regulation, genomic imprinting and the silencing of repetitive DNA elements, which occurs predominantly within $\mathrm{CpG}$ sites (4-6). $\mathrm{CpG}$ sites are underrepresented in the mammalian genome and tend to be clustered within $\mathrm{CpG}$ islands (CGIs) located in the vicinity of the transcription start sites (TSSs) of the majority of the human protein-coding genes (7). Inhibiting DNA methylation with cytidine analogues, including decitabine (DAC), reactivated the expression of genes, which were aberrantly silenced by hypermethylation. DAC is integrated into the replicating DNA 
and forms irreversible covalent bonds with the active site of DNA methyltransferase (8).

DAC was demonstrated to be active in several hematological disorders, including myelodysplastic syndrome (MDS), acute myelogenous leukemia (AML), chronic myelogenous leukemia (CML) and sickle cell anemia (9-13). Numerous previous studies in cancer cells demonstrated that DAC inhibits cell proliferation and the motility of the cells, and it is clinically administered as an antitumor drug to promote the expression of tumor suppressor genes (8,9,12-15). However, since demethylation across the entire genome alters the expression of a large number of genes, the effects of DAC in different tumor cell types are difficult to accurately predict $(16,17)$.

Neural precursor cell-expressed, developmentally downregulated (NEDD)4 is a prominent member of the E3-ubiquitin ligase family. NEDD4-1, a member of the NEDD4 subfamily, has a catalytic HECT domain at the C-terminus, and C2 and WW domains at the N-terminus, which are responsible for substrate recognition $(18,19)$. NEDD4-1 was reported to regulate a number of cellular functions, including the development of the neuromuscular junction (20) and the central nervous system, and axon guidance $(21,22)$, in addition to exerting a role in brain diseases $(23,24)$. From the perspective of studying cancer, NEDD4-1 is highly expressed in a wide range of tumor types, including colorectal cancer, bladder cancer and gastric carcinoma, and it was demonstrated to activate the phosphoinositide 3-kinase/Akt pathway through the degradation of phosphatase and tensin homolog (PTEN) (25-27). NEDD4-1 also promoted the migration and invasion of glioma cells via the ubiquitination and subsequent degradation of cyclic nucleotide-Ras guanine nucleotide exchange factors (CNrasGEFs) (28). In a major type of gastric cancer, gastric cardia adenocarcinoma, NEDD4-1 was revealed to be an exceptional prognostic biomarker (29).

In the present study, the effects of DAC in promoting the invasion and migration of MGC803 gastric cancer cells, or in inhibiting their proliferation, were investigated. Furthermore, the present study aimed to investigate the mechanistic role of NEDD4-1 in the invasion-promoting activity of DAC in the MGC803 cells, and whether the underlying mechanism involved CGI methylation, either in the NEDD4 promoter or in the first intron. The effects of DAC on different gastric cancer cell lines were subsequently discussed, including the therapeutic potential of DAC in clinical applications.

\section{Materials and methods}

Materials. A primary antibody against NEDD4 [cat. no. 2740; rabbit phospho-antibody (pAb); 1:500] was obtained from Cell Signaling Technology, Inc. (Danvers, MA, USA), and an antibody against $\beta$-actin [cat. no. sc-47778; horseradish peroxidase (HRP)-conjugated; 1:500] was purchased from Santa Cruz Biotechnology, Inc. (Santa Cruz, CA, USA). HRP-conjugated anti-rabbit/mouse secondary antibodies (A16072 and A16104) were obtained from Thermo Fisher Scientific (Waltham, MA, USA). Antibody against CNrasGEF (cat. no. WH0009693M1; mouse pAb; 1:400) and DAC (5-Aza-2'-deoxycytidine; cat. no A3656) were obtained from Sigma-Aldrich (St. Louis, MO, USA).
The pair of independent anti-NEDD4-1 stealth RNA interference (RNAi) small interfering (si)RNAs used in the present study, siN4-1 (5'-GAGTTCTTACAAGTGTGCAAA CAAA-3') and siN4-2 (5'-CCGATTGACAAGAGATGATTT CCTA-3'), Lipofectamine ${ }^{\circledR}$ RNAiMAX reagent, Opti-MEM ${ }^{\circledR}$ reduced serum media, Gibco fetal bovine serum (FBS) and SYBR $^{\circledR}$ Select Master mix were obtained from Thermo Fisher Scientific. HyClone ${ }^{\mathrm{TM}}$ RPMI-1640 medium was also obtained from Thermo Fisher Scientific. The PrimeScript ${ }^{\circledR}$ reverse transcription (RT) reagent kit with genomic (g)DNA Eraser was obtained from Takara Biotechnology Co., Ltd. (Dalian, China).

Cell culture and transfection. The human gastric cancer cell lines, MGC803, SGC7901 and NCI-N87, were purchased from the Cell Resource Center, IBMS, CAMS/PUMC (Beijing, China). The cell lines were maintained in either RPMI-1640 (SGC7901, NCI-N87) or in Dulbecco's modified Eagle's medium (MGC803; Gibco; Thermo Fisher Scientific), supplemented with $10 \% \mathrm{FBS}$ and $100 \mathrm{U} / \mathrm{ml}$ penicillin/streptomycin (HyClone ${ }^{\mathrm{TM}}$; Thermo Fisher Scientific) at $37^{\circ} \mathrm{C}$ in a humidified atmosphere $\left(5 \% \mathrm{CO}_{2} / 95 \%\right.$ air).

The siRNA transfections were performed according to the manufacturer's protocols in 6-well plates, using Lipofectamine ${ }^{\circledR}$ RNAiMAX transfection reagent.

Cell proliferation analysis. MGC803 or SGC7901 cells $\left(2 \times 10^{5}\right.$ cells $/ \mathrm{ml}$ in 96 -well plates) were treated with phosphate-buffered saline(PBS) or $1 \mu \mathrm{MDAC}$. The cell viability was measured using a 3-(4,5-dimethyl-2-thiazolyl)-2,5-diphenyl-2-tetrazolium bromide (MTT) cell viability detection kit (Beyotime Institute of Biotechnology, Beijing, China), according to the manufacturer's protocols.

The absorbance was measured at $490 \mathrm{~nm}$ using a microplate spectrophotometer (Spectra Max M3; Molecular Devices, LLC, Sunnyvale, CA, USA).

RNA extraction and RT-quantitative polymerase chain reaction $(q P C R)$. For the $\mathrm{RT}-\mathrm{qPCR}$ analyses, the total RNA was extracted from cells using Invitrogen TRIzol ${ }^{\circledR}$ reagent (Thermo Fisher Scientific), according to the manufacturer's protocols. Clearance of the DNA contamination in RNA and cDNA synthesis were performed using the PrimeScript ${ }^{\circledR}$ RT reagent kit with gDNA Eraser, according to the manufacturer's protocols (Takara Biotechnology Co., Ltd.). RT-qPCR was subsequently performed using the ABI-7500 system (Applied Biosystems; Thermo Fisher Scientific), using SYBR ${ }^{\circledR}$ Select Master mix (Thermo Fisher Scientific) according to the manufacturer's instructions. Primer sequences were as follows: NEDD4-1, forward (F): 5'-GGTGGAGGTGTTCGGGCT-3' and reverse (R): 5'-GCAAGGCCTATTCCGGCTA-3'; glyceraldehyde-3-phosphate dehydrogenase (GAPDH), F: 5'-GAG TCAACGGATTTGGTCGT-3' and R: 5'-GACAAGCTTCCC GTTCTCAG-3'.

Western blot analysis. The preparation of the whole cell lysates and western blot analysis were performed, as previously described (30). In brief, cells were harvested and washed in ice-cold PBS, then the protein concentration of the extracts was determined using bicinchoninic acid reagent (Thermo 
A
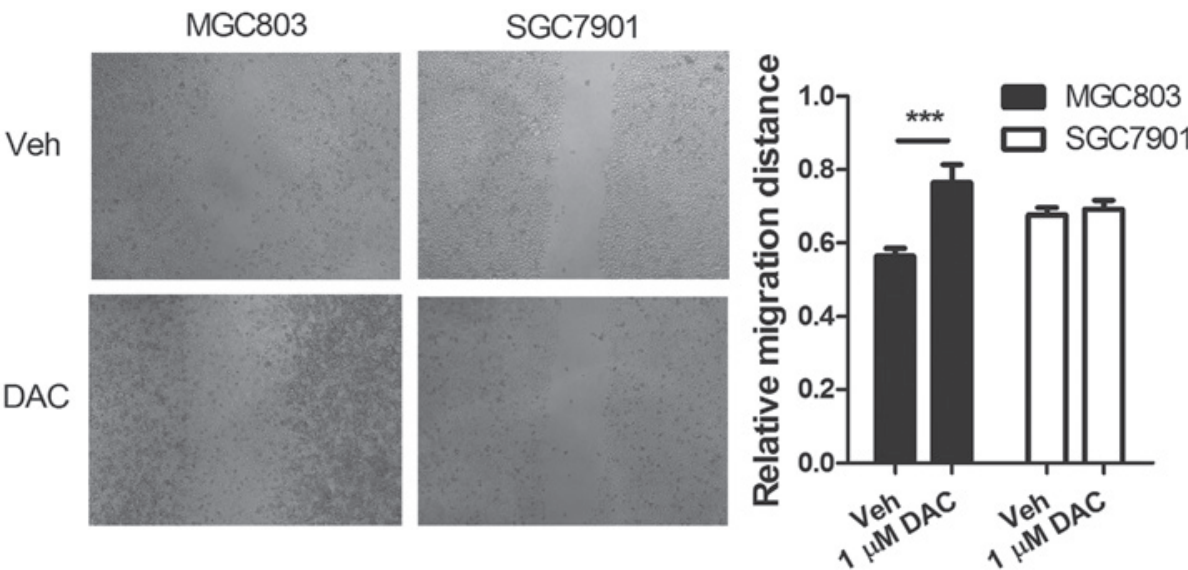

B

MGC803
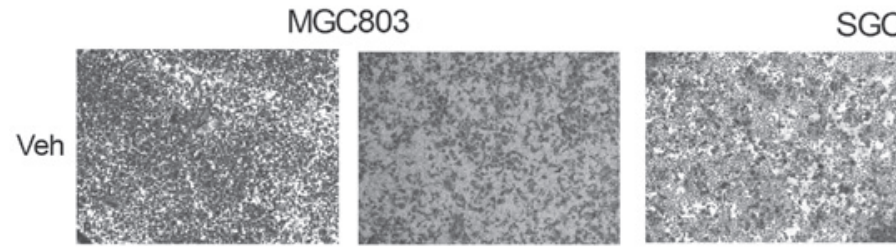

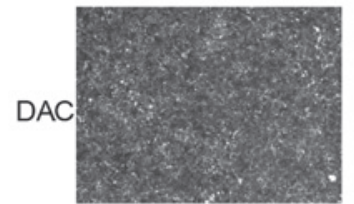

Migration

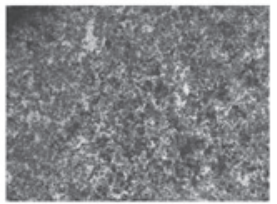

Invasion

C

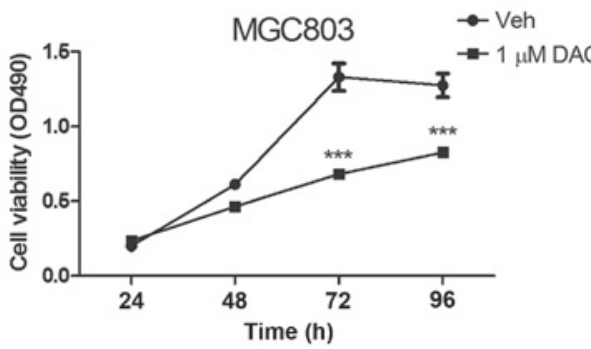

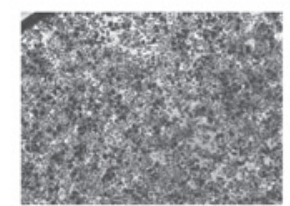

Migration
SGC7901
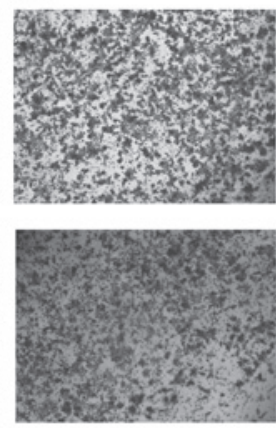

Invasion

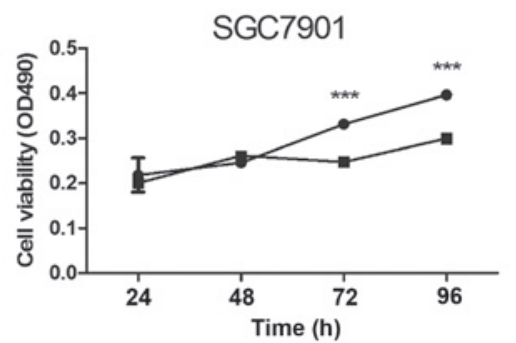

Figure 1. DNA methylation inhibitor, DAC, promotes MGC803 cell invasion and migration. (A) A wound-healing assay, and (B) a Transwell migration and invasion assay of the MGC03 and SGC7901 cells was performed following treatment with $1 \mu \mathrm{M} \mathrm{DAC} \mathrm{for} 48 \mathrm{~h}$; magnification, $\mathrm{x} 40$. (C) The viability of the MGC03 and SGC7901 cells was assessed following treatment with $1 \mu \mathrm{M}$ DAC or phosphate-buffered saline, as measured using a 3-(4,5-dimethyl-2-thiazolyl)-2,5-diphenyl-2-tetrazolium bromide assay. The data are expressed as the mean \pm standard deviation $\left(\mathrm{n}>3 ;{ }^{*} \mathrm{P}<0.05,{ }^{* *} \mathrm{P}<0.01\right.$ and ${ }^{* * *} \mathrm{P}<0.001$, compared with Veh) DAC, decitabine; OD, optical density; Veh, vehicle.

Fisher Scientific). Equal quantities of protein (30 g/lane) were loaded, separated using $12 \%$ SDS-PAGE and transferred onto nitrocellulose membranes. Subsequent to being blocked with $5 \%$ non-fat milk, membranes were incubated with the primary antibodies at $4^{\circ} \mathrm{C}$, overnight. Subsequent to incubation with the respective secondary antibody, immune complexes were detected using ECL western blotting reagents (Thermo Fisher Scientific). The expression levels of $\beta$-actin were monitored as the internal control, and band intensities were normalized to that of $\beta$-actin.

Wound healing assay. The cells were seeded into 6-well plates $\left(5 \times 10^{5}\right.$ cells) and were incubated until they had reached $90 \%$ confluence. At $24 \mathrm{~h}$ post-transfection, a vertical wound was created using a $200 \mu \mathrm{l}$ pipette tip. Subsequently, the cells were washed three times with PBS and medium without serum was added into the wells. Following a $48 \mathrm{~h}$ incubation with
$1 \mu \mathrm{M}$ DAC, the wound was observed and random fields in each well were selected for imaging. The captured images were analyzed using Image ${ }^{\circledR}$ software (National Institutes of Health, Bethesda, MD, USA), and the distance of the wound closure was used to estimate the migrational capacity of the cells.

Migration and invasion assay. The cell migrational and invasive abilities were determined using 24-well Transwell plates. For the migration assay, $24 \mathrm{~h}$ following transfection, $5 \times 10^{4}$ cells $/$ well were seeded into the top chamber and maintained in serum-free medium. Medium (600 $\mu \mathrm{l})$ containing $10 \%$ FBS was added into the bottom chamber. Following an incubation for $48 \mathrm{~h}$ at $37^{\circ} \mathrm{C}$, the cells which had migrated through the pore polycarbonate membrane were fixed with methanol and stained with Giemsa (Sigma-Aldrich). Subsequently, the cells that had migrated were observed and 
A

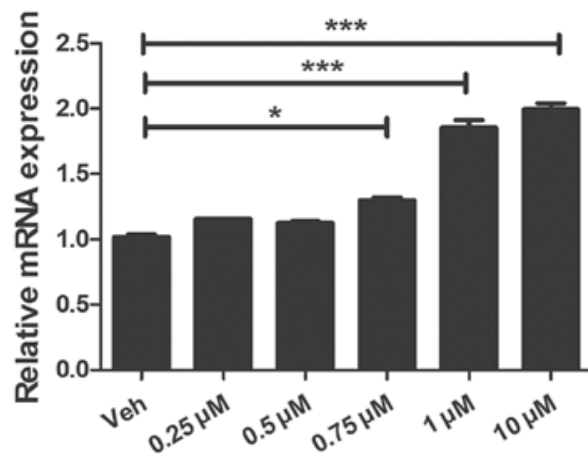

B

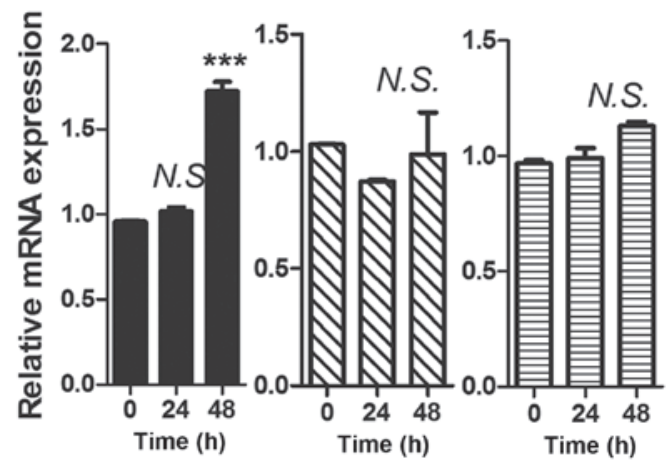

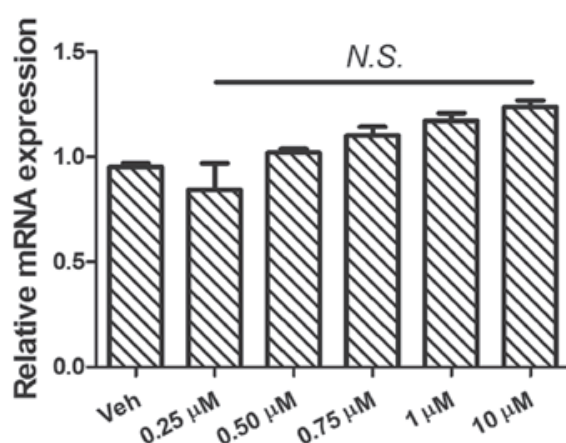

C

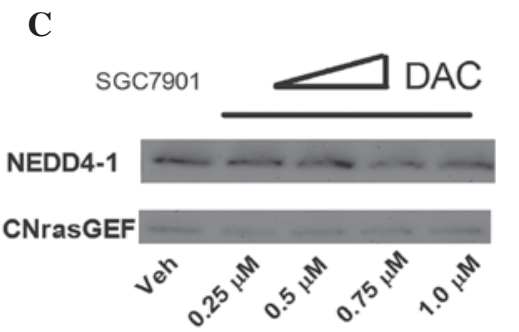

D
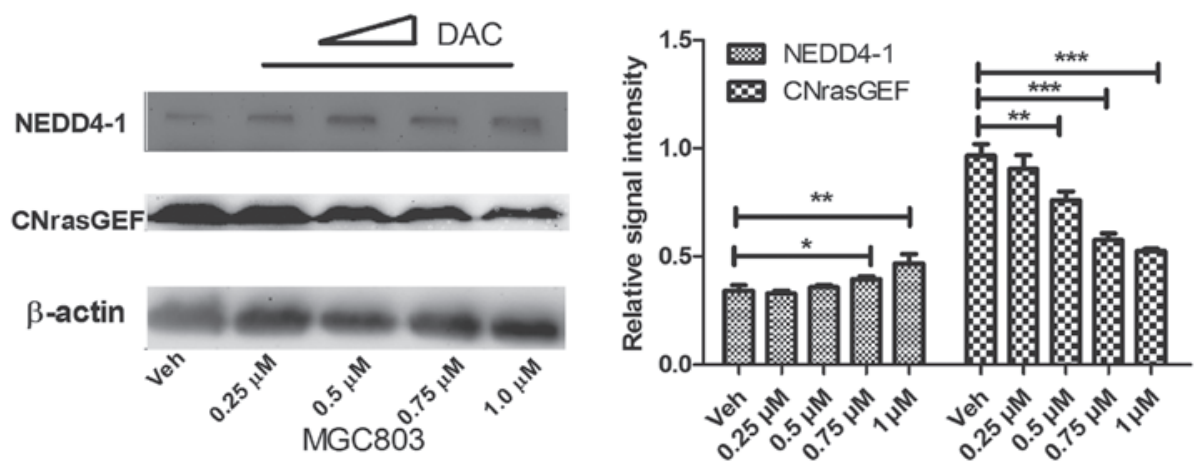

Figure 2. DAC promotes the expression of NEDD4-1 in MGC803 cells. (A) The mRNA expression of NEDD4-1 following an incubation with DAC for 48 h at the concentrations indicated in the figure is shown (left, MGC803; right, SGC7901). (B) The mRNA expression of NEDD4-1 following an incubation with $1 \mu \mathrm{M}$ DAC for 24 or $48 \mathrm{~h}$ is shown (left, MGC803; middle, SGC7901; right, NCI-N87). Representative western blots illustrating the protein expression levels of NEDD4-1 and CNrasGEF in (C) SGC7901 and (D) MGC803 cells, following an incubation with DAC at the indicated concentrations for 48 h. The bar-chart in (D) shows the quantification of the data illustrated in the western blot for the MGC 803 cells. The data are expressed as the mean \pm standard deviation ( $>3$ ). (A) Left bar-chart, $\mathrm{P}<0.05$; right bar-chart, $\mathrm{P}>0.05$ (one-way analysis of variance), compared with Veh; (B and $\mathrm{D}){ }^{*} \mathrm{P}<0.05{ }^{* * *} \mathrm{P}<0.01$; ${ }^{* * *} \mathrm{P}<0.001(\mathrm{Student} \mathrm{s}$ t-test), compared with time $0 \mathrm{~h}$ or Veh. DAC, decitabine; Veh, vehicle; CNrasGEF, cyclic nucleotide-Ras guanine nucleotide exchange factor; NEDD4-1, neural precursor cell-expressed, developmentally downregulated 4-1; N.S., not significant.

images were captured using microscopy (IX70; Olympus Corporation, Tokyo, Japan). For the invasion assay, prior to cell seeding, the Matrigel was diluted in serum-free medium to a final concentration of $1 \mathrm{mg} / \mathrm{ml}$. Diluted Matrigel (100 $\mu \mathrm{l} /$ well) was added into the top chamber and incubated for $4 \mathrm{~h}$ at $37^{\circ} \mathrm{C}$, followed by the identical procedures as described for the migration assay.

Bisulfite sequencing analysis. Bisulfite sequencing PCR (BS-PCR) was performed with gDNA extracted from MGC803, SGC7901 and NCI-N87 cells using an EpiTect ${ }^{\circledR}$ Fast LyseAll Bisulfite kit (Qiagen, Hilden, Germany). PCR reactions were performed using EpiTaq HS (Takara Biotechnology Co., Ltd.) with primers as follows: NEDD4 promoter, F: 5'-TTGTAG TGTTTTTTAGTAATAAGTTT-3' and R: 5'-TCTTATAAA
AATAACACCCTTAAC-'3; NEDD4 first intron, F: 5'-TTT TTTTTAATATTTTTTGAAGGAAATTG-3' and R: 5'-AAA ACTCTACTATCAACCCCTCCT-3'.

The PCR products were subcloned using a pMD-19 T vector (Takara Biotechnology Co., Ltd.), according to the manufacturer's protocols, and individual clones were subsequently sequenced (Sangon Biotech Co. Ltd., Shanghai, China). Clones were only accepted if at least $90 \%$ cytosine conversion occurred and all possible clonalities were excluded based on the criteria included in the BiQ Analyzer software (Max Planck Society, Munich, Germany). At least 10 replicates were performed for each of the selected regions in each cell line.

Statistical analysis. Each experiment was repeated at least in triplicate. Statistical analyses (Student's t-test or one-way 
A

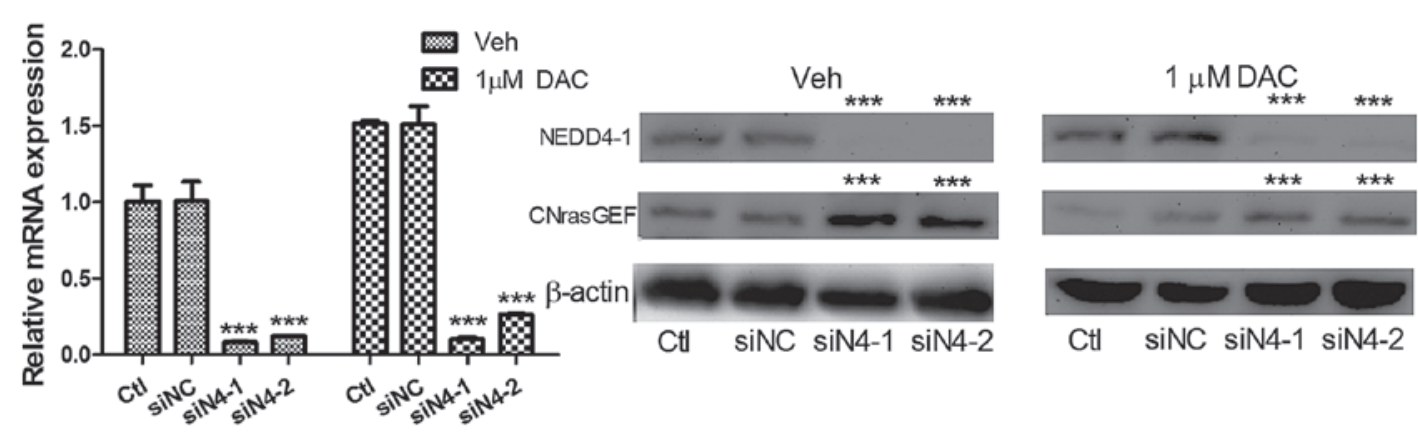

B
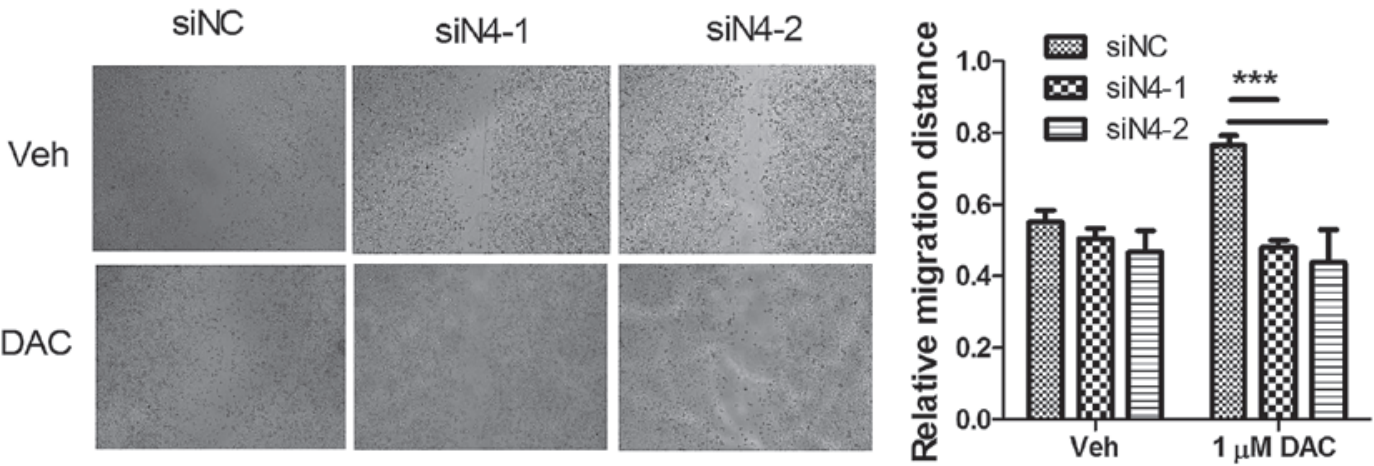

C
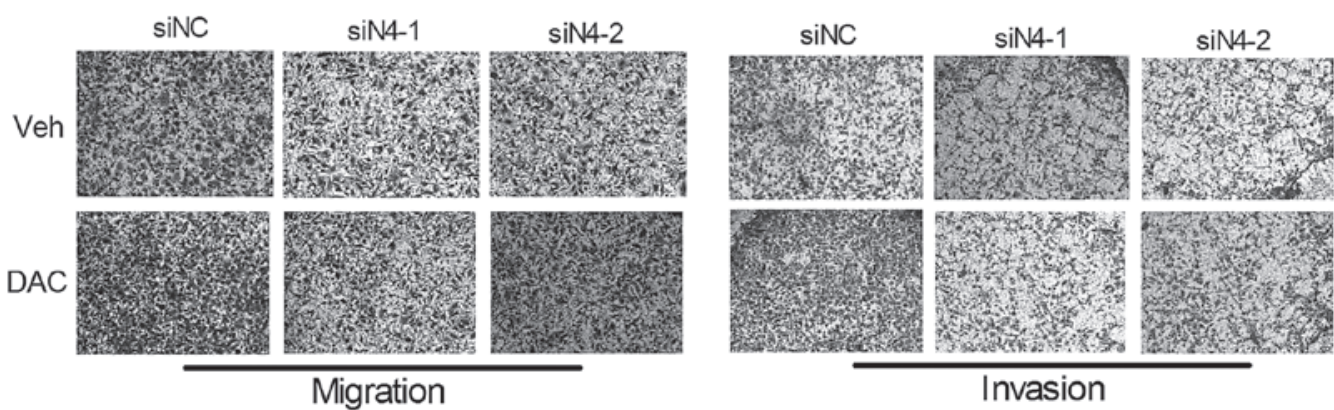

Figure 3. DAC promotes cell-invasive behavior in an NEDD4-1-dependent manner. (A) The mRNA and protein expression levels of the MGC03 cells following transfection with $50 \mathrm{nM}$ anti-NEDD4-1 siRNAs, and subsequent treatment with $1 \mu \mathrm{M}$ DAC/Veh for $48 \mathrm{~h}$. (B) The wound-healing assay of the MGC03 cells following transfection with $50 \mathrm{nM}$ anti-NEDD4-1 siRNAs, and subsequent treatment with $1 \mu \mathrm{M}$ DAC/Veh for $48 \mathrm{~h}$; magnification, x40. (C) The Transwell migration and invasion assays of the MGC03 cells following transfection with $50 \mathrm{nM}$ anti-NEDD4-1 siRNAs, and subsequent treatment with $1 \mu \mathrm{M}$ DAC/Veh for $48 \mathrm{~h}$; magnification, $\mathrm{x} 40$. The data are expressed as the mean \pm standard deviation $\left(\mathrm{n}>3 ;{ }^{*} \mathrm{P}<0.05,{ }^{* *} \mathrm{P}<0.01\right.$ and ${ }^{* * * *} \mathrm{P}<0.001$, compared with siNC). CNrasGEF, cyclic nucleotide-Ras guanine nucleotide exchange factor; Ctl, control; DAC, decitabine; NEDD4-1, neural precursor cell-expressed, developmentally downregulated 4-1; siNC, small interfering RNA negative control; Veh, vehicle.

analysis of variance) were performed using Microsoft Excel and statistical add-on software (Microsoft Corporation, Redmond, WA, USA). $\mathrm{P}<0.05$ was considered to indicate a statistically significant difference and the results are expressed as the mean \pm standard deviation.

\section{Results}

Decitabine promotes the invasion and migration of MGC803 gastric cancer cell lines. To examine the effect of DAC on gastric cancer cell invasion, wound-healing (Fig. 1A) and Transwell (Fig. 1B) assays were performed. The results revealed that $1 \mu \mathrm{M}$ DAC significantly promoted the invasion and migration of the MGC803 cells, although it failed to elicit a response in the SGC7901 gastric cancer cell line. Consistently with previously published studies $(31,32)$, DAC inhibited the proliferation of each cell line, without any marked difference in its effectiveness (Fig. 1C).
DAC promotes the expression of NEDD4-1 in MGC803 cells. Since NEDD4-1 has been demonstrated to be involved in glioma cell invasion (28), it was hypothesized that NEDD4-1 may also mediate the DAC-promoted invasion of the MGC803 gastric cancer cell line. At $48 \mathrm{~h}$ post-incubation with a dilute concentration of DAC, NEDD4-1 was upregulated in the MGC803 cells, and in agreement with the hypothesis, NEDD4-1 was not upregulated in the SGC7901 cells in the presence of DAC (Fig. 2A). Treatment with $1 \mu \mathrm{M}$ DAC for $24 \mathrm{~h}$ failed to elicit any expression of NEDD4-1 in the MGC803 cell line (Fig. 2B, left), perhaps as a consequence of the action of DAC-inhibiting DNA methyltransferases. Furthermore, treatment with $1 \mu \mathrm{M}$ DAC for 24 or $48 \mathrm{~h}$ failed to upregulate the expression of NEDD4-1 in either the SGC7901 or the NCI-N87 cell line (Fig. 2B, middle and right, respectively). Subsequent to the RT-qPCR analysis, DAC failed to induce the protein expression of NEDD4-1 in the SGC7901 cells (Fig. 2C). However, following an incubation for $48 \mathrm{~h}$ with a 
A

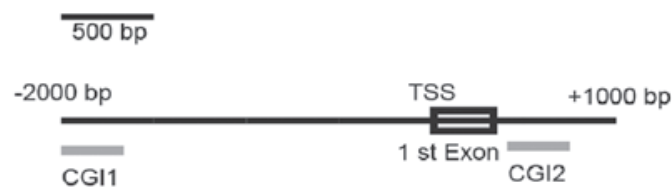

B

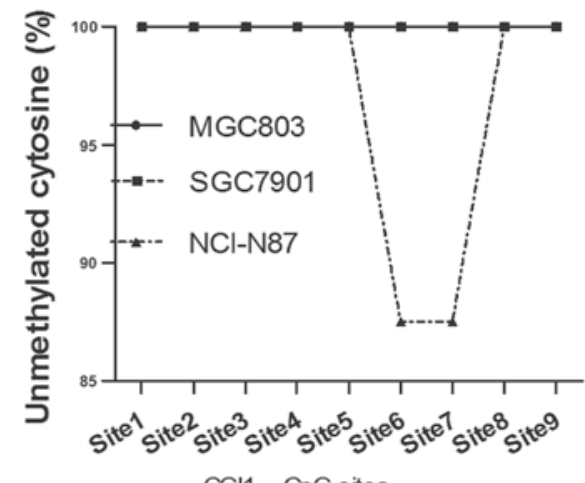

C

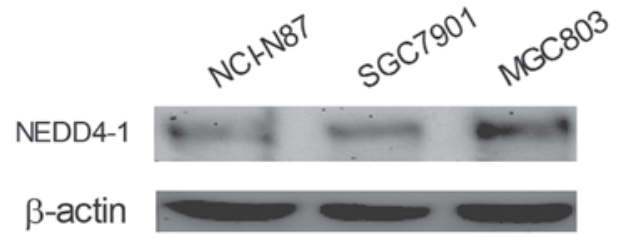

Figure 4. Methylation of CGI1 and CGI2 in the NEDD4-1 promoter of the first intron is not involved in the DAC-promoted expression of NEDD4-1. (A) A schematic of the structure of the NEDD4-1 promoter region, highlighting the locations of the CGIs. (B) The methylation of CGI1 in the upstream promoter region of NEDD4-1. (C) The protein expression level of NEDD4-1 in the NCI-N87, SGC7901 and MGC803 cell lines. bp, base pairs; CGI, CpG island; NEDD, neural precursor cell-expressed, developmentally downregulated; TSS: transcription start site.

dilute concentration of DAC, the protein expression level of NEDD4-1 was upregulated in the MGC803 cells, whereas CNrasGEF, which has been demonstrated to be a substrate of NEDD4-1, was downregulated (Fig. 2D) (28).

DAC promotes the cell-invasive behavior in an NEDD4-1-dependent manner. To determine whether the expression of NEDD4-1 was associated with the DAC-promoted cellular invasive properties of the MGC803 cells, the siRNA silencing of NEDD4-1 was investigated. The results from the RT-qPCR and western blotting experiments suggested that the siRNAs (siN4-1 and siN4-2) almost completely inhibited the mRNA and the protein expression levels of NEDD4-1 without DAC incubation (Fig. 3A). Following transfection with the NEDD4-1 siRNAs, the migratory ability of the cells on exposure to DAC was inhibited completely, although in the vehicle-treated group, knocking down the NEDD4-1 protein failed to disturb the cell migration rate in the wound healing assay (Fig. 3B). Furthermore, the Transwell migration and invasion assays exhibited a similar trend (Fig. 3C).

DAC-mediated upregulation of NEDD4-1 is not directly associated with the inhibition of methylation in CGIs in the first intron of the NEDD4-1 promoter. To further examine whether DAC promoted the expression of NEDD4-1 through the complete inhibition of DNA methylation, CGIs in the NEDD4-1 promoter and its first intron were initially investigated using MethPrimer (Fig. 4A) (33). There are two CGIs, CGI1 and 2, which are located in the promoter and in the first intron, respectively. Subsequently, BS-PCR was performed to measure the methylated cytosine content.

The results revealed that no clear DNA methylation existed in the CGIs of the NEDD4-1 promoter (Fig. 4B) or the first intron $(100 \%$ unmethylated cytosine identified in $23 \mathrm{CpG}$ sites). These data indicated that the DAC-promoted NEDD4-1 expression was not mediated by any direct alteration of the NEDD4-1 promoter or of intron methylation. Among the NCI-N87, SGC7901 and MGC803 cell lines, the expression of NEDD4-1 revealed no correlation with its methylation level (Fig. 4C).

\section{Discussion}

DNA methylation is a crucial epigenetic signature that is implicated in transcriptional regulation, which occurs predominantly within $\mathrm{CpG}$ sites $(3,4)$. CpG sites are underrepresented in the mammalian genome and tend to be clustered within CGIs located in the vicinity of the TSSs of the majority of the human protein-coding genes (5). The inhibition of DNA methylation using cytidine analogues, including DAC, reactivates the expression of genes, which were aberrantly silenced by hypermethylation. DAC integrates itself into replicating DNA, thereby forming irreversible covalent bonds with the active sites of DNA methyltransferase (6).

DAC was demonstrated to have activity in hematological disorders, including MDS, AML, CML and sickle cell anemia (9-13). Since it is administered in the clinic as an antitumor agent, DAC functions by completely inhibiting the proliferation and the motility of the cells $(8,9,12-15)$. However, since demethylation in the genome may alter the gene expression of a large number of genes, the effect of DAC in different tumor cell types remained controversial $(16,17)$.

In the present study, it was demonstrated for the first time, to the best of our knowledge, that DAC may promote cell-invasive behavior in a well-defined gastric cancer cell line, MGC803, whereas it revealed no affect on the invasive properties of another cell line, SGC7901. (Figs. 1A and B), However, in both cell lines, DAC inhibited cell proliferation (Fig. 1C). During the incubation with DAC, an already proven cancer-associated gene, NEDD4-1, was demonstrated to be upregulated in the MGC803 cells. NEDD4-1 exerts its activity as an E3-ubiquitin ligase, belonging to the NEDD4 family (24). Previous reports indicated that NEDD4-1 promotes glioma cell migration and invasion via the ubiquitination and degradation of CNrasGEFs (28). The results of the present study also suggested that DAC promotes the invasion of MGC803 cells via the upregulation of the expression of NEDD4-1 and by restricting the expression of CNrasGEFs. The MGC803, SGC7901 and NCI-N87 cell lines exhibited a marked difference in their aggressive behavior $(M>S>N)(29)$, and the expression levels of NEDD4-1 correlated with this trend (Fig. 4C). Knocking down the NEDD4-1 protein completely inhibited the invasion-promoting effect of DAC, however, notably, the cell behavior upon treatment with vehicle caused 
no change (Figs. 3B and C). These results collectively suggested that the constitutive expression of NEDD4-1 may not be a key factor in the aggressive behavior exhibited by MGC803 cells. Furthermore, no clear discernible differences in the mRNA (data not shown) and protein (Fig. 4C) expression levels were observed in these three cell lines, and similarly, no marked differences were observed in the DNA methylation status of the CGIs in the promoter and the first intron. It is therefore considered that DAC affects the expression level of NEDD4-1 in a DNA-methylation-independent manner, and the expression of NEDD4-1 in gastric cell lines may not be predominantly controlled by methylation.

Although PTEN was confirmed to be a NEDD4-1 substrate and it inhibits tumor cell proliferation, the upregulation of NEDD4-1 was incapable of promoting cell proliferation (Fig. 1C). This result may be accounted for by one of two hypotheses: (i) With the exception of NEDD4-1, DAC may alter the expression of other genes, including proliferation-associated genes; (ii) Other pathways are responsible for regulating PTEN, independently of NEDD4-1. In the present study, three commercial antibodies of PTEN were examined, none of which proved capable of successfully detecting the expression of PTEN in the MGC803, SGC7901 or the NCI-N87 cell lines.

Taken together, the present study suggested that DAC promoted cell-invasive behavior in the MGC803 gastric cancer cell line by upregulating the level of NEDD4-1 and restricting the level of CNrasGEFs. However, cell proliferation was inhibited, a result which was consistent with previous studies performed on other cancer cell lines $(34,35)$. Furthermore, CPI methylation in neither the NEDD4 promoter nor the first intron was observed. This study has revealed that DAC exerts different effects in different gastric cancer cell lines, which may provide a novel aspect for the consideration of clinical applications of DAC in the future.

\section{Acknowledgements}

The authors would like to thank other members of the $\mathrm{Yu}$ laboratory for a critical assessment of the manuscript. The present study was supported by grants from the health department of Heilongjiang Province (no. 2012-669), the education department of Heilongjiang Province (no. 12531270), and a grant from the National Natural Science Foundation of China (no. 81172417).

\section{References}

1. Melton SD, Genta RM and Souza RF: Biomarkers and molecular diagnosis of gastrointestinal and pancreatic neoplasms. Nat Rev Gastroenterol Hepatol 7: 620-628, 2010.

2. Correa P and Houghton J: Carcinogenesis of Helicobacter pylori. Gastroenterology 133: 659-672, 2007.

3. Macdonald JS, Smalley SR, Benedetti J, Hundahl SA, Estes NC, Stemmermann GN, Haller DG, Ajani JA, Gunderson LL, Jessup JM and Martenson JA: Chemoradiotherapy after surgery compared with surgery alone for adenocarcinoma of the stomach or gastroesophageal junction. N Engl J Med 345: 725-730, 2001

4. Jones PA and Baylin SB: The fundamental role of epigenetic events in cancer. Nat Rev Genet 3: 415-428, 2002.

5. Robertson KD: DNA methylation, methyltransferases and cancer. Oncogene 20: 3139-3155, 2001.

6. Guo M and Yan W: Epigenetics of gastric cancer. Methods Mol Biol 1238: 783-799, 2015
7. Illingworth RS, Gruenewald-Schneider U, Webb S, Kerr AR James KD, Turner DJ, Smith C, Harrison DJ, Andrews R and Bird AP: Orphan $\mathrm{CpG}$ islands identify numerous conserved promoters in the mammalian genome. PLoS Genet 6: e1001134, 2010.

8. Nie J, Liu L, Li X and Han W: Decitabine, a new star in epigenetic therapy: The clinical application and biological mechanism in solid tumors. Cancer Lett 354: 12-20, 2014.

9. Kantarjian H, Issa JP, Rosenfeld CS, Bennett JM, Albitar M, DiPersio J, Klimek V, Slack J, de Castro C, Ravandi F, et al: Decitabine improves patient outcomes in myelodysplastic syndromes: Results of a phase III randomized study. Cancer 106: 1794-1803, 2006.

10. Issa JP, Garcia-Manero G, Giles FJ, Mannari R, Thomas D, Faderl S, Bayar E, Lyons J, Rosenfeld CS, Cortes J and Kantarjian HM: Phase 1 study of low-dose prolonged exposure schedules of the hypomethylating agent 5-aza-2'-deoxycytidine (decitabine) in hematopoietic malignancies. Blood 103: 1635-1640, 2004.

11. Sacchi S, Kantarjian HM, O'Brien S, Cortes J, Rios MB, Giles FJ, Beran M, Koller CA, Keating MJ and Talpaz M: Chronic myelogenous leukemia in nonlymphoid blastic phase: Analysis of the results of first salvage therapy with three different treatment approaches for 162 patients. Cancer 86: 2632-2641, 1999.

12. Kantarjian HM, O'Brien S, Cortes J, Giles FJ, Faderl S, Issa JP, Garcia-Manero G, Rios MB, Shan J, Andreeff M, et al: Results of decitabine (5-aza-2'deoxycytidine) therapy in 130 patients with chronic myelogenous leukemia. Cancer 98: 522-528, 2003.

13. Lubbert M, Suciu S, Baila L, Rüter BH, Platzbecker U, Giagounidis A, Selleslag D, Labar B, Germing U, Salih HR, et al: Low-dose decitabine versus best supportive care in elderly patients with intermediate- or high-risk myelodysplastic syndrome (MDS) ineligible for intensive chemotherapy: Final results of the randomized phaseIII study of the European organisation for research and treatment of cancer Leukemia group and the German MDS study group. J Clin Oncol 29: 1987-1996, 2011.

14. Shin DY, Kim GY, Kim CG, Kim WJ, Kang HS and Choi YH: Anti-invasive effects of decitabine, a DNA methyltransferase inhibitor, through tightening of tight junctions and inhibition of matrix metalloproteinase activities in AGS human gastric carcinoma cells. Oncol Rep 28: 1043-1050, 2012.

15. Liu WH, Sang MX, Hou SY, Zhang C and Shan BE: Low-dose decitabine induces MAGE-A expression and inhibits invasion via suppression of NF- $\mathrm{kB} 2$ and MMP2 in Eca109 cells. Biomed Pharmacother 68: 745-750, 2014.

16. Jiao F, Bai SY, Ma Y, Yan ZH, Yue Z, Yu Y, Wang X and Wang J: DNA methylation of heparanase promoter influences its expression and associated with the progression of human breast cancer. PLoS One 9: e92190, 2014.

17. Borges S, Döppler HR and Storz P: A combination treatment with DNA methyltransferase inhibitors and suramin decreases invasiveness of breast cancer cells. Breast Cancer Res Treat 144: 79-91, 2014.

18. Rougier JS, Albesa M, Abriel H and Viard P: Neuronal precursor cell-expressed developmentally down-regulated 4-1 (NEDD4-1) controls the sorting of newly synthesized $\mathrm{Ca}(\mathrm{V}) 1.2$ calcium channels. J Biol Chem 286: 8829-8838, 2011.

19. Kwak YD, Wang B, Pan W, Xu H, Jiang X and Liao FF: Functional interaction of phosphatase and tensin homologue (PTEN) with the E3 ligase NEDD4-1 during neuronal response to zinc. J Biol Chem 285: 9847-9857, 2010.

20. Liu Y, Oppenheim RW, Sugiura Y and Lin W: Abnormal development of the neuromuscular junction in Nedd4-deficient mice. Dev Biol 330: 153-166, 2009.

21. Kawabe H, Neeb A, Dimova K, Young SM Jr, Takeda M, Katsurabayashi S, Mitkovski M, Malakhova OA, Zhang DE, Umikawa M, et al: Regulation of Rap2A by the ubiquitin ligase Nedd4-1 controls neurite development. Neuron 65: 358-372, 2010.

22. Kawabe $\mathrm{H}$ and Brose N: The ubiquitin E3 ligase Nedd4-1 controls neurite development. Cell Cycle 9: 2477-2478, 2010.

23. Lackovic J, Howitt J, Callaway JK, Silke J, Bartlett P and Tan SS: Differential regulation of Nedd4 ubiquitin ligases and their adaptor protein Ndfip1 in a rat model of ischemic stroke. Exp Neurol 235: 326-335, 2012.

24. Kwak YD, Wang B, Li JJ, Wang R, Deng Q, Diao S, Chen Y, $\mathrm{Xu} \mathrm{R}$, Masliah E, Xu H, et al: Upregulation of the E3 ligase NEDD4-1 by oxidative stress degrades IGF-1 receptor protein in neurodegeneration. J Neurosci 32: 10971-10981, 2012. 
25. Wang X, Trotman LC, Koppie T, Alimonti A, Chen Z, Gao Z, Wang J, Erdjument-Bromage H, Tempst P, Cordon-Cardo C, et al: NEDD4-1 is a proto-oncogenic ubiquitin ligase for PTEN. Cell 128: 129-139, 2007.

26. Amodio N, Scrima M, Palaia L, Salman AN, Quintiero A, Franco R, Botti G, Pirozzi P, Rocco G, De Rosa N and Viglietto G: Oncogenic role of the E3 ubiquitin ligase NEDD4-1, a PTEN negative regulator, in non-small-cell lung carcinomas. Am J Pathol 177: 2622-2634, 2010.

27. Kim SS, Yoo NJ, Jeong EG, Kim MS and Lee SH: Expression of NEDD4-1, a PTEN regulator, in gastric and colorectal carcinomas. APMIS 116: 779-784, 2008.

28. Zhang H, Nie W, Zhang X, Zhang G, Li Z, Wu H, Shi Q, Chen Y, Ding Z, Zhou $X$ and Yu R: NEDD4-1 regulates migration and invasion of glioma cells through CNrasGEF ubiquitination in vitro. PLoS One 8: e82789, 2013.

29. Sun A, Yu G, Dou X, Yan X, Yang W and Lin Q: Nedd4-1 is an exceptional prognostic biomarker for gastric cardia adenocarcinoma and functionally associated with metastasis. Mol Cancer 13: 248, 2014.
30. Lin F, Lin P, Zhao D, Chen Y, Xiao L, Qin W, Li D, Chen H, Zhao B, Zou H, et al: Sox 2 targets cyclinE, p27 and survivin to regulate androgen-independent human prostate cancer cell proliferation and apoptosis. Cell Prolif 45: 207-216, 2012.

31. Wang X, Wang H, Jiang N, Lu W, Zhang XF and Fang JY: Effect of inhibition of MEK pathway on 5-aza-deoxycytidine-suppressed pancreatic cancer cell proliferation. Genet Mol Res 12: 5560-5573, 2013.

32. Wang B, Li H, Yang R, Zhou S and Zou S: Decitabine inhibits the cell growth of cholangiocarcinoma in cultured cell lines and mouse xenografts. Oncol Lett 8: 1919-1924, 2014.

33. Li LC and Dahiya R: MethPrimer: Designing primers for methylation PCRs. Bioinformatics 18: 1427-1431, 2002.

34. Everson RG, Antonios JP, Lisiero DN, Soto H, Scharnweber R, Garrett MC, Yong WH, Li N, Li G, Kruse CA, et al: Efficacy of systemic adoptive transfer immunotherapy targeting NY-ESO-1 for glioblastoma. Neuro Oncol: Sep 1, 2015 (Epub ahead of print).

35. Yan W, Herman JG and Guo M: Epigenome-based personalized medicine in human cancer. Epigenomics: Sep 7, 2015 (Epub ahead of print). 